\title{
Hand Sanitizing Within the College Settings
}

\author{
Mayra Alvarez ${ }^{\mathrm{a}}$, Hollyanne George ${ }^{\mathrm{a}}$, Kathryn Wheeler ${ }^{\mathrm{a}}$, Kathy Sexton Radek ${ }^{\mathrm{a}}$
}

The purpose of this paper is to evaluate, educate and promote the importance of hand sanitizing within the Elmhurst College community. We are looking to provide insight into the impact hand sanitizer plays in the everyday activities of our students. Our proposed findings would indicate that increased usage of hand sanitizing with the use of hand sanitizer would lower the chances of illness, illness spreading and doctor visits associated with poor hand hygiene.

Hand hygiene is one of the most imperative hygiene practices. When we think about hand hygiene, we typically associate it with using soap and water. However, within the fast-paced environment we live in, hand sanitizing plays an essential role in hands hygiene. Hand sanitizing is not only more readily available, but it is cost effective and cheaper than a visit to the doctor's office from contracting a preventable illness or infection. In specific circumstances where soap and water is not accessible such as camping or outdoor activities, hand sanitizer would be the ideal alternative to hand washing.

\section{Health Promoting Behaviors}

The importance of using hand sanitizer can be correlated to the education and informative measures along with the readily available supplies on hand within the college environment. Improving hand hygiene has proven to be an effective method in decreasing infection rates (Dollinger, Gaube, Lemer, Tsirvrikos, 2018). The theory of planned behavior is most widely applicable to hand sanitizing. The driving forces behind the usage of hand sanitizer is the intent to perform the action. An example of this is the idea or notion that one does not need to practice hand hygiene and will be immune to illness by building up their resistance to illness by exposure. The intent is predicted and driven by three forces, which include attitude- knowledge and belief about hand sanitizing and the outcomes. In addition, subjective norms within our society leads to one's own view on how we view hand sanitizing but also how others view it as well. Finally, due to perceived behavior control or our ability to perform the act of cleansing our hands is based on our own personal beliefs. The planned behavior theory determines the framework for adherence to recommended hand hygiene practices.

\section{Proposal}

Our proposal for the usage of hand sanitizing and the use of hand sanitizer within Elmhurst College includes a sampling of our incoming freshmen and transfer students prior to their first semester. We intend to disburse "Bluejay Welcome Pack" to each incoming student during orientation. We also intend to give additional packs to faculty and staff within the community of the college. The packs will include small, pocket-sized hand sanitizer, Band-Aids, anti-bacterial ointment, amongst other health necessities. Our aim is to analyze how effective hand-sanitizer is and how many times students are utilizing our wellness center for illness that could have been prevented with the use of hand sanitizing. Our independent variable is the use of hand sanitizer and the dependent variable is the measure of the effectiveness of the hand sanitizer within the semester used. In order to analyze our findings, we would work with the wellness center to relay a number of wellness checks/ illness exams performed within each month over the course of a semester. The proposed findings would be that there would be a decreased finding of preventable illnesses reported due to the readily available usage of hand sanitizer provided.

\section{Implications}

By analyzing our findings for one semester, we can further evaluate whether or not the usage of hand sanitizer would be beneficial to use for future semesters and whether or not it is preventing illness from spreading. To understand the importance of using hand hygiene we need to also evaluate whether or not our students are informed on how illness can be prevented and how easily it spreads across a college campus. Research suggests hand hygiene products need to be available; people need to know when to properly perform hand hygiene measures and need to be within an environment that promotes or encourages the use of hand sanitizing (Dollinger, Gaube, Lemer, Tsirvrikos, 2018). An additional measure that we think would be beneficial to the use of hand sanitizer is monthly reminders of emails that elaborates on the importance of sanitizing ones hands within the changing of seasons and when illness is at its peak.

\section{References}

Gaube S, Tsivrikos D, Dollinger D, Lermer E (2018). How a smiley protects health: A pilot intervention to improve hand hygiene in hospitals by activating injunctive norms through emotions. PloS ONE 13(5): e0197465.

https://www.researchgate.net/publication/325279214_How_a_smiley_protects_health_A_pilot_intervention_to_improve_hand_h ygiene_in_hospitals_by_activating_injunctive_norms_through_emoticons 